\title{
Outcomes of the Pediatric Development Plan of Tapentadol
}

This article was published in the following Dove Press journal: Journal of Pain Research

\author{
Mariëlle Eerdekens (1D \\ Tatjana Radic (D) \\ Melanie Sohns \\ Feras Khalil (D) \\ Beata Bulawa (D) \\ Christian Elling $\mathbb{D}$ \\ Grünenthal GmbH, Aachen, Germany
}

\begin{abstract}
The opioid analgesic tapentadol was the first pain medication to be developed for the treatment of pain in children under a formal process established by the regulatory authorities. This article summarizes the outcomes of the pediatric development program for tapentadol across the entire age range from birth (including neonates) to adolescents $<18$ years of age. In addition, the challenges experienced when designing and conducting the pediatric tapentadol clinical trials as well as the interactions with the regulatory authorities are discussed. As a first outcome, the oral solution of tapentadol was authorized in the EU in 2018 as a new treatment option in the hospital setting for moderate to severe acute pain in children from 2 to $<18$ years of age.
\end{abstract}

Keywords: pain, pediatric, regulations, tapentadol, review

\section{Introduction}

Very few pain medications are authorized for pediatric use, in particular for the neonatal and infant population. ${ }^{1}$ The treatment of acute or long-term pediatric pain often relies on data extrapolated from adult trials and best practice guidelines recommended by global experts. ${ }^{2}$ However, children are not simply small adults; this vulnerable population requires medications thoroughly assessed for their pharmacokinetic (PK) and pharmacodynamic (ie safety and efficacy) properties across a wide age and developmental range. In order to support the development of medicines for children, both the European Medicines Agency (EMA) and the US Food and Drug Administration (FDA) established a regulatory framework ${ }^{3,4}$ includ- $^{-}$ ing the Pediatric Study Plan (US) and the Pediatric Investigation Plan (EU). These are mandatory unless a waiver or deferral has been granted, and incentives are available if particular conditions are fulfilled. Currently, incentives for the pharmaceutical industry to conduct these trials include exclusive marketing for 6 months in the US and a 6-month extension of the supplementary protection certificate in the EU.

Not all medications in development for adults may be suitable for use in the pediatric population. Specific conditions for which some medications are developed may not occur in children, or a product may not offer significant therapeutic benefits or may be considered ineffective or unsafe. Moreover, for pain medications with an opioid mechanism of action, their potential for developing an opioid use disorder needs to be considered. ${ }^{5}$ For tapentadol, an opioid analgesic with two mechanisms of action, it was clear that a development program in the pediatric population was appropriate as pain is present in children and the treatment may be a suitable option for this population.
Correspondence: Mariëlle Eerdekens Grünenthal GmbH, Zieglerstraße 6, 52078 Aachen, Germany

Tel +49 24I 569 I090

Email marielle.eerdekens@grunenthal. com 
Tapentadol was shown to provide effective pain relief in multiple acute and chronic pain indications in adult patients ${ }^{6-8}$ with a more favorable gastrointestinal tolerability profile compared to typical opioids acting through $\mu$ receptors only. This profile can be explained by the combination of two synergistic mechanisms of action in one molecule ie $\mu$-opioid receptor agonism (MOR) and noradrenaline reuptake inhibition (NRI) ${ }^{9}$ resulting in a reduced $\mu$-load. ${ }^{9,10}$ The investigation of tapentadol for the relief of moderate to severe pediatric pain was not only supported by its benefit/ risk profile in adults but also by its specific pharmacokinetic and pharmacogenomic profile ${ }^{5}$ resulting in a predictable PK profile $^{11}$ and the absence of active metabolites contributing to the analgesic effect. ${ }^{12}$ With Phase II glucuronidation as the main metabolic pathway, ${ }^{13}$ there is a low potential for drugdrug interactions. ${ }^{14,15}$

These considerations have led to an agreed pediatric investigational plan with the Pediatric Committee of the EMA (PDCO) in the EU and an agreed pediatric study plan with the US FDA for the development of various tapentadol formulations.

\section{Efficacy and Safety of Tapentadol in the Pediatric Population}

An overview of the trials included in the tapentadol pediatric program is shown in Figure 1; Table 1 provides a summary of the trials and main trial outcomes. The guiding principle for the pediatric tapentadol program was that similar exposure in children as observed in adults at effective dose levels would result in an effective and safe profile in children. Therefore, extensive PK profiling supported by modelling and simulation techniques took place to address all age groups and all tapentadol formulations used in this program and to determine the doses used in the efficacy trials. ${ }^{16,17}$

\section{Efficacy}

Treatment of Acute Pain in the Pediatric Population The single-dose PK trials were powered to assess the PK profile of tapentadol oral solution (OS) and tapentadol intravenous infusion (IV) in the pediatric population in order to determine appropriate tapentadol doses for the treatment of acute pain in children. Efficacy evaluations were thus only exploratory but permitted a first indication about the effectiveness of the medication. Pain intensity was measured using ageappropriate rating scales. In older children ( $\geq 2$ years), mean pain intensity improved during the first hours after tapentadol OS administration. ${ }^{18,19}$ Supplemental analgesic medications were given on average at least $5 \mathrm{~h}$ after tapentadol administration which coincided with a decrease in tapentadol serum concentrations. In children $<2$ years, mean pain intensity reductions were already observed $15 \mathrm{~min}$ after the start of tapentadol OS or IV treatment. ${ }^{20}$

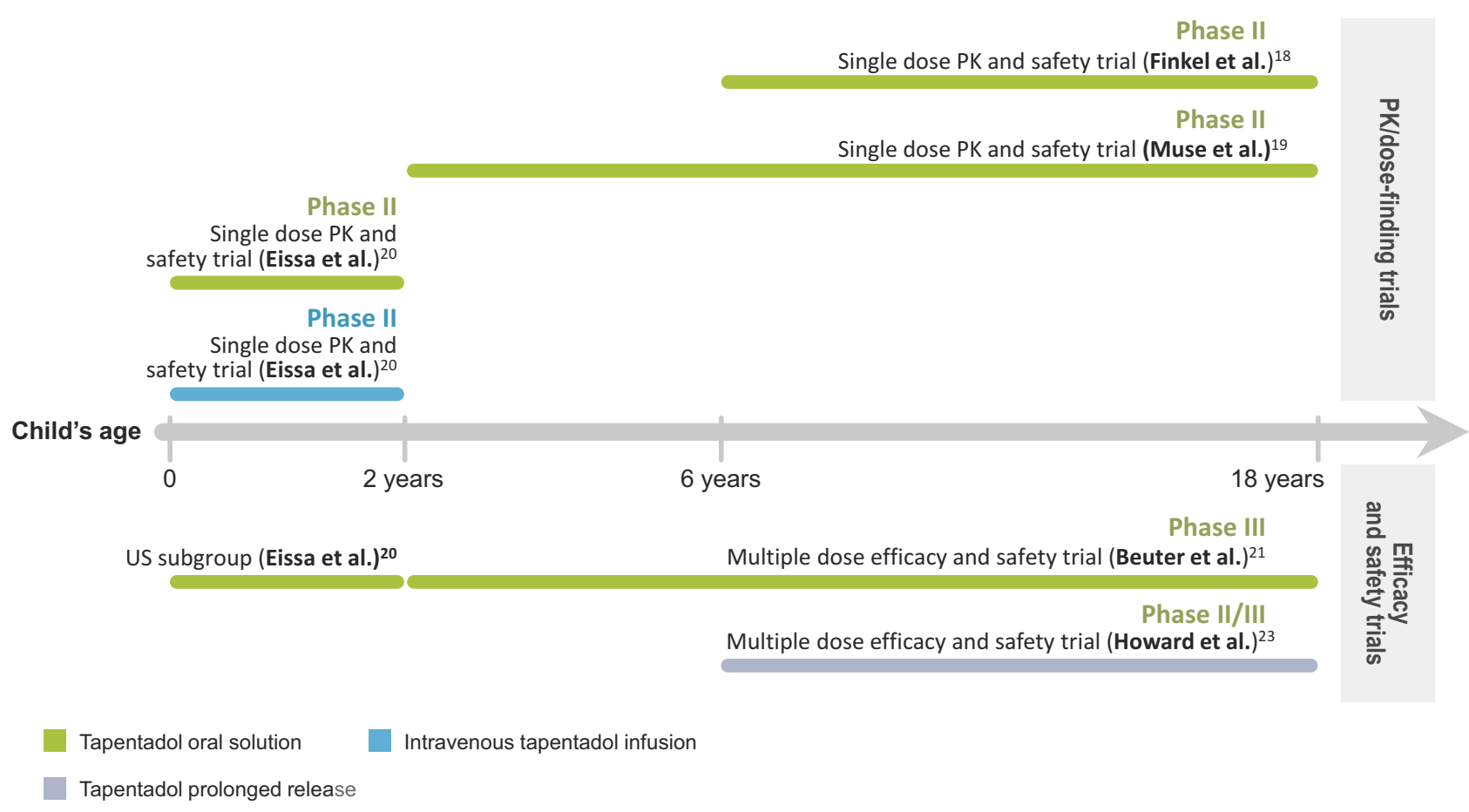

Figure I Overview of the trials included in the tapentadol developmental program. 
Table I Summary of Tapentadol Trials for Moderate to Severe Acute or Long-Term Pediatric Pain

\begin{tabular}{|c|c|c|c|}
\hline Trial & Pharmacokinetics & Efficacy & Safety \\
\hline \multicolumn{4}{|l|}{ Acute pain } \\
\hline $\begin{array}{l}\text { Single dose PK trial (Finkel } \\
\text { et al) }{ }^{18} \\
\text { Open-label, multicenter, phase } 2 \\
6 \text { to }<18 \text { years, } \mathrm{n}=44 \\
\text { I } \mathrm{mg} / \mathrm{kg} \text { bodyweight tapentadol } \\
\text { OS }\end{array}$ & $\begin{array}{l}\text { Tapentadol OS I mg/kg provided } \\
\text { serum concentrations comparable } \\
\text { to therapeutic levels in adults }\end{array}$ & $\begin{array}{l}\text { Exploratory analysis } \\
\text { Gradual reductions in mean pain } \\
\text { intensity scores from baseline } \\
\text { during the first } 4 \mathrm{~h} \text { following } \\
\text { tapentadol administration; use of } \\
\text { supplemental analgesics on } \\
\text { average started approx. } 5 \mathrm{~h} \text { after } \\
\text { tapentadol administration } \\
\text { consistent with a decrease in } \\
\text { tapentadol serum concentrations }\end{array}$ & $\begin{array}{l}\text { TEAEs (none severe) in } 45.5 \% \text { of } \\
\text { patients, most commonly vomiting } \\
(29.5 \%) \text { and nausea }(9.1 \%) \\
\text { No serious TEAEs occurred }\end{array}$ \\
\hline $\begin{array}{l}\text { Single dose } \mathbf{P K} \text { trial (Muse } \\
\text { et al) }{ }^{19} \\
\text { Open-label, single center, phase } 2 \\
2 \text { to }<18 \text { years, } \mathrm{n}=66 \\
\text { I } \mathrm{mg} / \mathrm{kg} \text { bodyweight tapentadol } \\
\text { OS }\end{array}$ & $\begin{array}{l}\text { Tapentadol OS I mg/kg provided } \\
\text { serum concentrations within the } \\
\text { targeted range shown to be safe } \\
\text { and efficacious in adults }(n=56)\end{array}$ & $\begin{array}{l}\text { Exploratory analysis } \\
\text { Mean pain intensity improved } \\
\text { Median time to first intake of } \\
\text { supplemental analgesics after } \\
\text { tapentadol administration was } 6.3 \mathrm{~h}\end{array}$ & $\begin{array}{l}\text { TEAEs (none severe) in } 57.6 \% \text { of } \\
\text { patients, most commonly nausea } \\
(24.2 \% \text { ) and vomiting ( } 16.7 \%) \\
\text { No serious TEAEs occurred }\end{array}$ \\
\hline $\begin{array}{l}\text { Single dose PK trial (Eissa } \\
\text { et al) } \\
\text { Open-label, multicenter, phase } 2 \\
\text { Birth to }<2 \text { years, } \mathrm{n}=19 \\
0.5-0.75 \mathrm{mg} / \mathrm{kg} \text { bodyweight } \\
\text { tapentadol OS }\end{array}$ & $\begin{array}{l}\text { Mean tapentadol serum } \\
\text { concentrations within or at the } \\
\text { lower end of the targeted range } \\
\text { known to be safe and efficacious } \\
\text { in adults; also compared well to } \\
\text { range observed in older children. } \\
(n=18)\end{array}$ & $\begin{array}{l}\text { Exploratory analysis } \\
\text { Mean pain intensity reductions } \\
\text { already observed } 15 \text { min after } \\
\text { start of tapentadol treatment } \\
94.7 \% \text { of patients received } \\
\text { concomitant analgesic medication }\end{array}$ & $\begin{array}{l}\text { TEAEs (one severe) in } 42.1 \% \text { of } \\
\text { patients, most commonly vomiting } \\
(10.5 \%) \\
\text { No serious TEAEs occurred }\end{array}$ \\
\hline $\begin{array}{l}\text { Single dose PK trial (Eissa } \\
\text { et al) } \\
\text { Open-label, multicenter, phase } 2 \\
\text { Preterm/term neonates to }<2 \\
\text { years, } \mathrm{n}=38 \\
0.3-0.4 \mathrm{mg} / \mathrm{kg} \text { bodyweight } \\
\text { tapentadol IV }\end{array}$ & $\begin{array}{l}\text { Tapentadol serum concentration } \\
\text { profile after IV administration } \\
\text { similar to that observed after } \\
\text { administration of tapentadol OS } \\
\text { across the age range (birth to }<18 \\
\text { years) }\end{array}$ & $\begin{array}{l}\text { Exploratory analysis } \\
\text { Mean pain intensity reductions } \\
\text { already observed } 15 \text { min after } \\
\text { start of tapentadol treatment } \\
86.6 \% \text { of patients received } \\
\text { concomitant analgesic medication }\end{array}$ & $\begin{array}{l}\text { TEAEs (two severe) in } 28.9 \% \text { of } \\
\text { patients; most commonly vomiting } \\
(10.5 \%) \\
\text { No serious TEAEs occurred }\end{array}$ \\
\hline \multicolumn{4}{|l|}{$\begin{array}{l}\text { Confirmatory multiple dose } \\
\text { efficacy and safety trial } \\
\text { Randomized, double-blind, } \\
\text { placebo-controlled, multicenter, } \\
\text { phase } 3 \text { with an immediate rescue } \\
\text { trial design }\end{array}$} \\
\hline $\begin{array}{l}2 \text { to }<18 \text { years } \\
\text { Tapentadol } n=108 \text {, placebo } n=52 \\
1.25 \mathrm{mg} / \mathrm{kg} \text { bodyweight tapentadol } \\
\text { OS (Beuter et al) }{ }^{21}\end{array}$ & nd & $\begin{array}{l}\text { The total amount of supplemental } \\
\text { opioid analgesic medication within } \\
\text { in the first } 24 \mathrm{~h} \text { after start of trial } \\
\text { medication was significantly lower } \\
\text { in tapentadol than placebo } \\
\text { patients ( } \mathrm{p}=0.0154 \text { ). Taste and } \\
\text { palatability of tapentadol OS were } \\
\text { well perceived by most patients. }\end{array}$ & $\begin{array}{l}\text { TEAEs in } 50 \% \text { of placebo and } \\
57.4 \% \text { of tapentadol patients, } \\
\text { most commonly vomiting, nausea } \\
\text { and constipation }\end{array}$ \\
\hline
\end{tabular}


Table I (Continued).

\begin{tabular}{|c|c|c|c|}
\hline Trial & Pharmacokinetics & Efficacy & Safety \\
\hline $\begin{array}{l}\text { Birth to }<2 \text { years } \\
\text { Tapentadol } \mathrm{n}=\mathrm{II} \text {, placebo } \mathrm{n}=4 \\
0 . \mathrm{I}-\mathrm{I} .25 \mathrm{mg} / \mathrm{kg} \text { bodyweight } \\
\text { tapentadol OS (Eissa et al) }\end{array}$ & nd & $\begin{array}{l}\text { The total amount of supplemental } \\
\text { opioid analgesic medication within } \\
\text { the first } 24 \mathrm{~h} \text { after start of trial } \\
\text { medication was low (placebo } \\
0.02 \mathrm{mg} / \mathrm{kg} \text {, tapentadol } 0.05 \mathrm{mg} / \\
\mathrm{kg} \text { ). Owing to the very low sample } \\
\text { size, the analysis was only } \\
\text { descriptive. } \\
\text { As expected, changes in mean } \\
\text { pain intensity were similar } \\
\text { between treatments. }\end{array}$ & $\begin{array}{l}\text { TEAEs in } 75 \% \text { of placebo and } \\
54.5 \% \text { of tapentadol patients } \\
\text { No serious TEAEs occurred } \\
\text { No indication of higher } \\
\text { susceptibility to adverse } \\
\text { tapentadol effects compared to } \\
\text { pediatric patients } \geq 2 \text { years }\end{array}$ \\
\hline \multicolumn{4}{|l|}{ Long-term pain } \\
\hline $\begin{array}{l}\text { Tapentadol PR pediatric trial } \\
\text { (Howard et al) }^{23} \\
\text { Open-label, I4-day randomized, } \\
\text { active-controlled (morphine PR), } \\
\text { multicenter, phase } 2 / 3 \text { with open- } \\
\text { label tapentadol PR extension up } \\
\text { to } 12 \text { months or no treatment } \\
\text { safety observation period; } n=69 \\
6 \text { to }<18 \text { years } \\
\text { I.25-I.5 mg/kg bodyweight } \\
\text { tapentadol PR every } 12 \mathrm{~h} \\
\text { Randomized phase: tapentadol PR } \\
\mathrm{n}=45 \text {, morphine PR } \mathrm{n}=24 \\
\text { Extension phase: } \mathrm{n}=36 \\
\text { Safety observation phase: } \mathrm{n}=58^{\mathrm{a}}\end{array}$ & $\begin{array}{l}\text { The pediatric population } \\
\text { estimation of accumulation of } 1.86 \\
\text { was in line with that obtained in } \\
\text { adults (I.4-I.7). }{ }^{36}\end{array}$ & $\begin{array}{l}\text { Randomized phase: } \\
\text { Tapentadol PR reduced pain } \\
\text { intensity from moderate to mild } \\
\text { pain and showed non-inferiority } \\
\text { to the active comparator } \\
\text { morphine PR based on response; } \\
\text { none of the patients discontinued } \\
\text { treatment due to lack of efficacy. } \\
\text { Rescue medication for } 40 \% \text { of } \\
\text { tapentadol PR, } 25 \% \text { of morphine } \\
\text { PR patients } \\
\text { Extension phase: } \\
\text { Pain intensity levels remained } \\
\text { stable }\end{array}$ & $\begin{array}{l}\text { Randomized phase: } \\
\text { TEAEs for } 57.8 \% \text { of tapentadol PR } \\
\text { and } 50 \% \text { of morphine PR patients } \\
\text { most commonly nausea ( } 22.2 \%) \\
\text { for tapentadol PR and vomiting } \\
(33.3 \%) \text { for morphine PR patients } \\
\text { Extension phase: } \\
\text { TEAEs in } 83.3 \% \text { of patients, most } \\
\text { commonly nausea ( } 30.6 \%) \text { and } \\
\text { headache }(27.8 \%) \\
\text { Safety observation phase: } \\
\text { None of the AEs were considered } \\
\text { long-term consequences of the } \\
\text { trial medication. }\end{array}$ \\
\hline
\end{tabular}

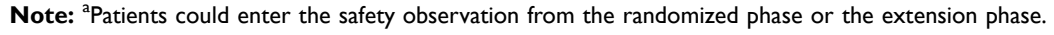

Abbreviations: AE, adverse event; IV, intravenous; nd, not determined; OS, oral solution; PK, pharmacokinetic; PR, prolonged release; TEAE, treatment-emergent adverse event.

The tapentadol OS doses established by PK analyses and subsequent population PK (popPK) modelling were investigated in a randomized, double-blind, placebocontrolled, multiple-dose confirmatory trial. This trial employed an immediate rescue design to ensure that all patients had access to pain medication (supplemental opioid analgesic medication) irrespective of their allocated treatment group. Efficacy in the treatment of moderate to severe acute pain was determined by comparing the amount of supplemental opioid analgesic medication used within the first $24 \mathrm{~h}$ after the first dose of trial medication in the tapentadol and the placebo groups. In children aged 2 to $<18$ years, tapentadol OS showed significantly better efficacy compared to placebo (Figure 2). ${ }^{21}$ The result was confirmed by sensitivity analyses and supported by secondary efficacy analyses. In the subgroup of children aged $<2$ years, the sample size was too low to allow for a comprehensive comparison of the treatment groups. ${ }^{20}$

The findings led to the approval of tapentadol OS in the EU for children 2 to $<18$ years of age for the treatment of moderate to severe acute pain which can be adequately managed only with opioid analgesics (dose recommendation of $1.25 \mathrm{mg} / \mathrm{kg}$ bodyweight every $4 \mathrm{~h}) .^{22}$ In line with the design of the clinical trials conducted in a hospital setting and with a total duration of 72 hours as agreed with the PDCO, the use of tapentadol OS is currently limited to a hospital setting and for up to 3 days only. Moreover, because in children below the age of 2 years only sparse efficacy and safety data and no PK data following multiple dosing are available, no tapentadol formulation is currently authorized for the treatment of pain in children $<2$ years of age. 


\section{Treatment of Long-Term Pain in the Pediatric Population}

An open-label, randomized, active-controlled tapentadol prolonged release (PR) trial with subsequent tapentadol extension phase investigated the efficacy of tapentadol PR in children aged 6 to $<18$ years suffering from a wide range of painful conditions expected to require opioid treatment for more than 14 days. ${ }^{23}$ Pain intensity was measured using ageappropriate rating scales. The primary efficacy endpoint was the number of treatment responders.

Tapentadol PR reduced pain intensity from moderate to mild pain and showed non-inferiority to the active comparator morphine PR over the 14-day randomized treatment period. Sensitivity analyses supported the primary endpoint result. None of the patients discontinued treatment due to lack of efficacy. For patients requiring long-term treatment pain intensity scores remained stable during the subsequent 12-month tapentadol extension phase (Figure 3).

In contrast to adults with chronic pain conditions who would generally only receive PR opioids after experiencing pain for at least 3 months and who usually continue on these medications for long periods, pediatric patients had a shorter history of pain and in most cases did not require pain treatment with a strong analgesic for a long period of time: nearly half of the patients entering the extension phase $(44.4 \%)$ discontinued treatment during the first 3 months, mainly because opioid treatment was no longer required. Although usually administered for a short period of time, a twice-daily dosing with a PR formulation might be a useful alternative to immediate release (IR) opioids in patients $\geq 6$ years as IR formulations require more frequent dosing (eg every 4-6 h) which may impact on patient compliance and may be difficult and time-consuming for caregivers as acknowledged by the FDA. $^{24}$

\section{Safety}

The tapentadol safety profile was as expected for an analgesic which includes an opioid mode of action. The adverse event profile also reflected specific characteristics of the pediatric trial population, its underlying diseases, and concomitant treatments. Compared to adults, there was no higher susceptibility of the pediatric trial population to side-effects. There were no new safety issues or tapentadol-related adverse events identified in pediatric trials.

\section{Tapentadol OS/IV}

Treatment-emergent adverse events (TEAEs) were reported for $51.2 \%$ of patients in single-dose tapentadol OS trials ${ }^{18-20}$ and for $28.9 \%$ of patients in the single-dose tapentadol IV trial; ${ }^{20}$ TEAEs occurred in $57.1 \%$ of patients receiving multiple doses of tapentadol OS for the treatment of acute pain ${ }^{20,21}$ (Table 2). None of the TEAEs in the single-dose trials were serious; two patients in the multidose trial, however, experienced serious TEAEs (abdominal abscess and seizure) ${ }^{21}$ but they were deemed unlikely related to tapentadol treatment by the investigators. The main TEAEs were vomiting (21.4\% [53/248]) and nausea (14.5\% [36/248]) across the tapentadol OS trials and vomiting $(10.5 \%)$ in the tapentadol IV trial. Of note is that pyrexia was reported in 5.2\% (15/286) of all patients dosed with tapentadol. This is not a known sideeffect of tapentadol treatment and none of these events were deemed related to trial medication by the investigators. The occurrence of pyrexia can probably be explained by confounding factors reported for these patients such as inflammation reactions due to surgery or an orthopedic implant, pre-existing fever, peri-splenitis, skin inflammation, or fever due to extensive blood loss. Incidences of pyrexia have also been reported under placebo, tapentadol, and oxycodone treatment for acute pain following major surgery in adult patients. ${ }^{7}$

Respiratory depression is a major clinical concern when administering centrally acting opioid analgesic medications. Respiratory depression was not documented as a TEAE in any of the trials. Furthermore, the incidence of TEAEs suggestive of respiratory depression was low: oxygen saturation decreased $(3.2 \%$ of patients $[9 / 286])$, hypoxia $\left(1.4 \%\right.$ [4/286]), and $\mathrm{PO}_{2}$ decreased $(0.4 \%$ [1/ 286]). Most of these TEAEs were considered not related to tapentadol treatment by the investigators; three cases of hypoxia were deemed at least possibly related.

Multiple doses of tapentadol OS were well tolerated across the age range. The proportions of patients with TEAEs were $54.5 \%$ (birth to $<2$ years), $39.1 \%$ ( 2 to $<6$ years), $46.9 \%$ ( 6 to $<12$ years), and $71.7 \%$ (12 to $<18$ years). Patients in the oldest age group received a higher amount of supplemental opioid analgesic medication than the other groups which might have contributed to the higher TEAE incidences.

There was no indication for a higher susceptibility to side-effects of the youngest patients $(<2$ years of age) compared to the older groups aged 2 years and above. In 


\section{LSMD (95\% CI) P value}

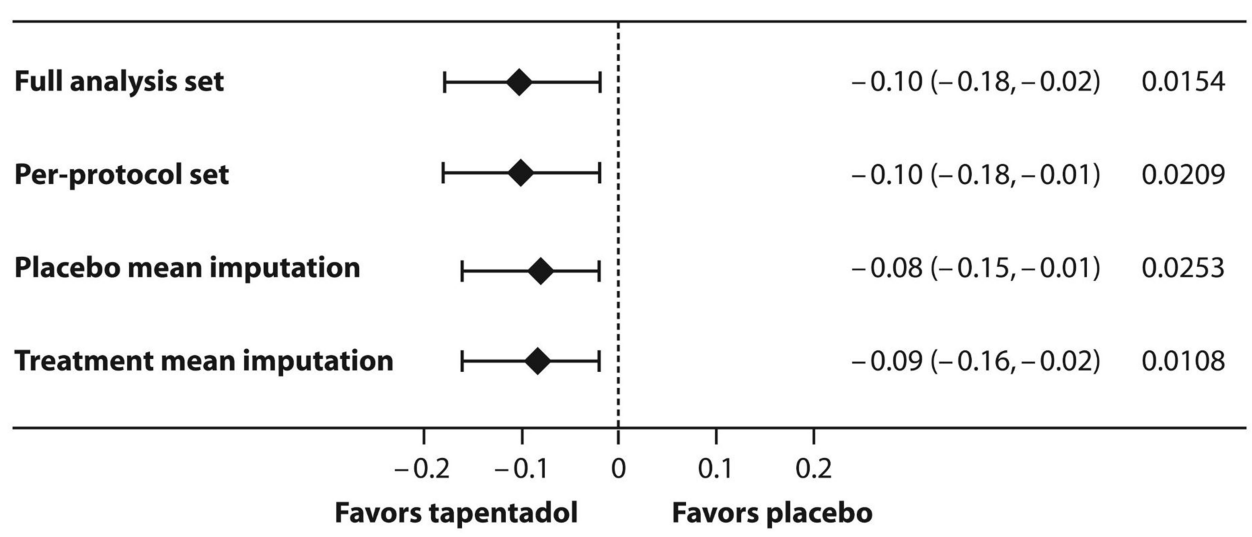

Figure 2 Statistically significant treatment differences in favor of tapentadol oral solution for the primary trial endpoint (amount of supplemental opioid analgesic medication used within the first $24 \mathrm{~h}$ after intake of trial medication) in children aged 2 to $<18$ years with moderate to severe acute pain. Main analysis (full analysis set) and sensitivity analyses.

Notes: Copyright 2019. Dove Medical Press. Reprinted from Beuter C, Volkers G, Radic T, Goldberg J, van den Anker J. Efficacy and safety of multiple doses of tapentadol oral solution in the treatment of moderate to severe acute pain in children aged 2 to $<18$ years - a randomized, double-blind, placebo-controlled trial. J Pain Res. 2019;12:3099-3112. ${ }^{21}$

Abbreviation: LSMD, least-squares mean difference.
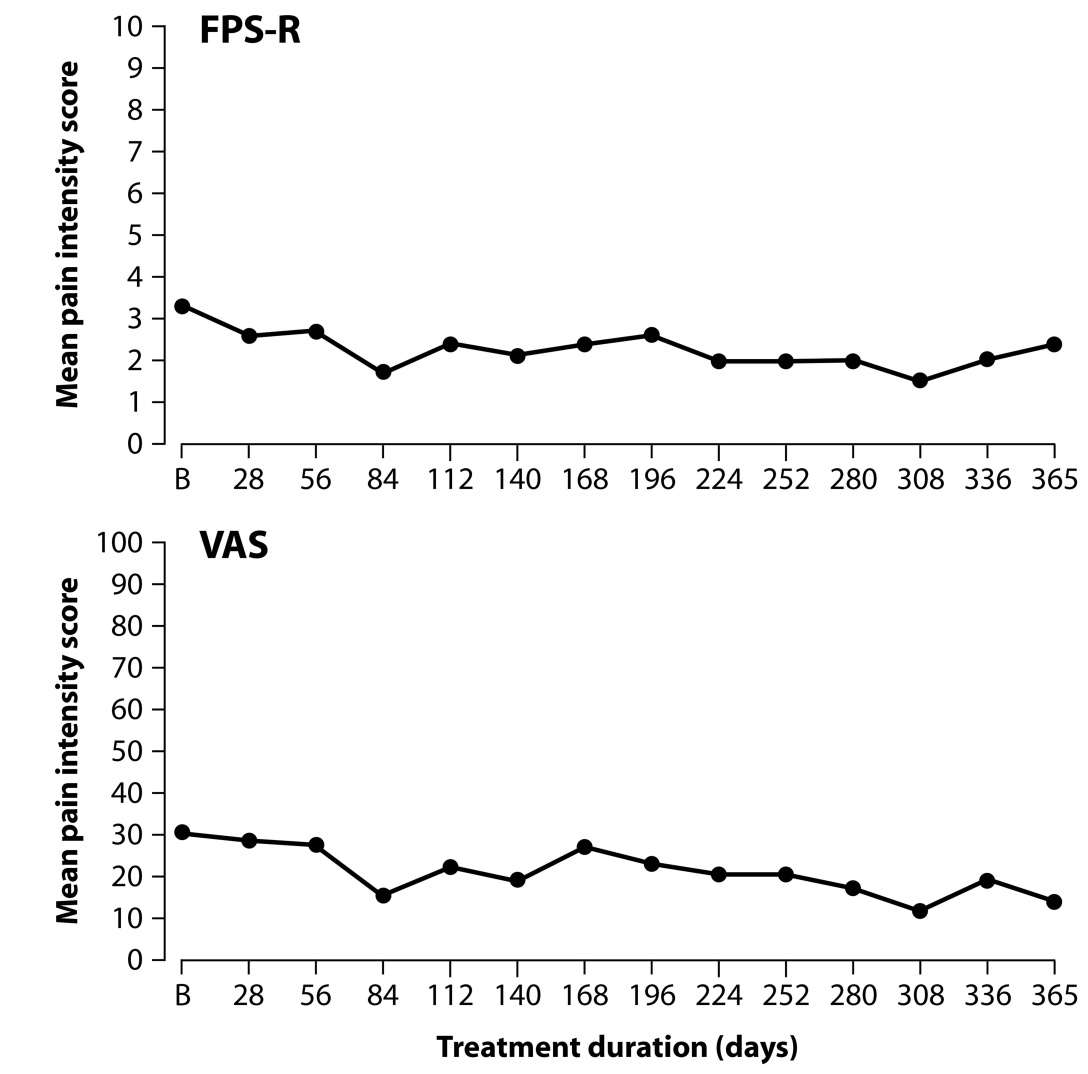

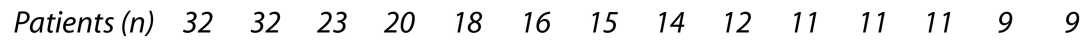

Figure 3 Stable pain intensity scores during up to 12 months treatment with tapentadol PR in children 6 to $<18$ years of age using two pain rating scales ("as observed" data).

Notes: Baseline was the last evaluation at or before the end of randomized treatment visit. Copyright 2020. Reprinted with permission from Howard R, Radic T, Sohns M, Eerdekens M, Waßmuth A. Tapentadol prolonged release for long-term treatment of pain in children. J Pain Res. 2020;13:3157-3170. ${ }^{23}$

Abbreviations: B, baseline; FPS-R, Faces Pain Scale-revised; VAS, visual analogue scale. 
Table 2 Main Treatment-Emergent Adverse Events under Tapentadol Treatment for Acute Pain (Including Events Suggestive of Respiratory Depression)

\begin{tabular}{|c|c|c|c|}
\hline & $\begin{array}{l}\text { OS Single Dose } \\
(n=\mid 29)\end{array}$ & $\begin{array}{l}\text { OS Multiple Doses } \\
(n=119)\end{array}$ & $\begin{array}{l}\text { IV Single Dose } \\
(n=38)\end{array}$ \\
\hline All patients with a TEAE & $66(51.2 \%)$ & $68(57.1 \%)$ & II (28.9\%) \\
\hline Vomiting & $26(20.2 \%)$ & $27(22.7 \%)$ & $4(10.5 \%)$ \\
\hline Nausea & $20(15.5 \%)$ & $16(13.4 \%)$ & 0 \\
\hline Dizziness & 7 (5.4\%) & $4(3.4 \%)$ & 0 \\
\hline Somnolence & $2(1.6 \%)$ & $6(5 \%)$ & 0 \\
\hline Constipation & I (0.8\%) & $12(10.1 \%)$ & 0 \\
\hline Pyrexia & $4(3.1 \%)$ & $10(8.4 \%)$ & I (2.6\%) \\
\hline Oxygen saturation decreased & $2(1.6 \%)$ & $4(3.4 \%)$ & $3(7.9 \%)$ \\
\hline Hypoxia & I (0.8\%) & $3(2.5 \%)$ & 0 \\
\hline $\mathrm{PO}_{2}$ decreased & 0 & I (0.8\%) & 0 \\
\hline
\end{tabular}

Notes: Data are number of patients (\%). Data from single-dose OS trials were combined.

Abbreviations: IV, intravenous; OS, oral solution; TEAE, treatment-emergent adverse event.

all age groups, vomiting was the most common TEAE: 2 patients $(18.2 \%)$ in the group $<2$ years, 4 patients $(17.4 \%)$ in the 2 to $<6$ years group, 8 patients $(25 \%)$ in the group from 6 to $<12$ years, and 13 patients $(24.5 \%)$ aged from 12 to $<18$ years.

Overall, there were no clinically relevant changes in hematology and clinical chemistry parameters under multiple-dose tapentadol OS treatment.

\section{Tapentadol PR}

Tapentadol PR treatment was well tolerated. The most frequently reported TEAEs were nausea $(22.2 \%$ of patients), constipation (15.6\%), and abdominal pain, vomiting and headache (each 13.3\%) during the 14-day randomized period and nausea $(30.6 \%)$ and headache $(27.8 \%)$ during the extension phase. The degree of constipation remained stable during the first 14 days; a slight decrease was observed under long-term treatment.

Small fluctuations in clinical chemistry and hematology laboratory parameters and in vital signs were not considered clinically relevant. There was one TEAE indicative of respiratory depression (bradypnea of moderate intensity) which was considered by the investigator to be certainly related to tapentadol PR treatment and led to treatment withdrawal. There was no indication of clinically relevant opioid withdrawal symptoms.

The adverse event profile was overall in line with the safety profile known from adult tapentadol PR trials; ${ }^{8}$ no new safety issues or tapentadol-related side-effects were identified. None of the adverse events occurring after the final tapentadol dose were considered to be long-term consequences of the trial medication.

\section{Inclusion of Results from the Development Plan into the Product Label}

In the US, the pediatric study plan is still ongoing. In the EU, the findings of the clinical trial program led to the approval of tapentadol OS for children 2 to $<18$ years of age for the treatment of moderate to severe acute pain which can be adequately managed only with opioid analgesics (dose recommendation of $1.25 \mathrm{mg} / \mathrm{kg}$ bodyweight every $4 \mathrm{~h}){ }^{22}$ The clinical program had been agreed with PDCO assuming that it would allow extension of the label from adults to children. This assumption turned out to be invalid. In contrast to the adult program, regulatory authorities approved a more restricted label in the pediatric population by mimicking the clinical trial conditions in the product label. As a result, tapentadol OS use is restricted to a hospital setting, limited to a total duration of use of 72 hours and use in children $\geq 2$ years of age as the available data from the agreed program with PDCO were deemed insufficient in the younger age group.

From this experience, it is clear that the requirements for approving a pediatric formulation might differ from the agreed development plan. The sufficiency of the data acquired for the PR formulation in children/adolescents is currently under re-evaluation prior to submission of a request for label extension of tapentadol PR to children $\geq 6$ years.

\section{New Insights into the Pediatric Development of Drugs Initially Targeted for Adults}

The challenges of conducting pediatric trials for the treatment of pain have been discussed in detail in the 
introductory article of the thematic tapentadol series. ${ }^{1}$ This chapter describes how some of these challenges were handled in the tapentadol program.

\section{Formulations Developed for Adult Patients May Not Be Suitable for Dosing Across the Entire Pediatric Age Range}

Tapentadol is available for adult patients as immediate release $^{25}$ and prolonged release $\mathrm{e}^{26}$ tablets and as an oral solution. ${ }^{22,27}$ Oral solutions are a common, generally wellaccepted formulation even for very young infants. However, to accommodate for weight-adjusted dosing, a lower OS dose strength had to be developed for younger children. In addition, an intravenous drug formulation for neonates and children unable to take oral medications was developed for the treatment of acute pain in the pediatric development program. Palatability and acceptability of the oral solution were investigated in a multiple-dose efficacy and safety trial in children aged 2 to $<18$ years $^{21}$ and were considered sufficient to ensure intake compliance in this age range. Conditions of chronic pain are rare in children and even more so in the very young; therefore, the PR formulation of tapentadol already approved for adult pain treatment was assessed for the treatment of pediatric pain in children aged 6 years and older requiring longer treatment.

\section{Determination of Age-Appropriate Analgesic Doses is Possible by Using popPK Modeling}

The determination of age-appropriate doses for the pediatric population can be based on a thorough understanding of the drug PK profile and its age-related changes. However, this thorough understanding might be difficult to achieve given the limitations in conducting pediatric trials. These limitations include the small sample size of pediatric clinical trials to minimize medication exposure in this vulnerable population and a restricted number of blood samples for PK analysis.

In order to maximize the information gain from the available samples, modelling and simulation techniques were extensively used in the tapentadol program.

In the development of tapentadol for the treatment of acute pain in pediatrics, a similar exposure-response relationship in children and adults was assumed, and the aim was therefore to identify tapentadol doses resulting in exposures in children which would fall into the adult exposure range using the approved therapeutic tapentadol IR dose range (50-100 $\mathrm{mg}$ ).

Throughout the tapentadol pediatric program, a staggered approach was adopted starting with exposing the oldest patients first even within the same trial. To best inform the dose selection, a popPK model that was developed on pooled data from adult trials of tapentadol IR was extended with allometric scaling to create the initial pediatric popPK model. This model was used to perform simulations and recommend doses to be investigated in the very first pediatric trials in subjects 2 years of age and above. ${ }^{18,19}$ As a result, a dose of $1.0 \mathrm{mg} / \mathrm{kg}$ was selected in order to match the targeted adult exposure.

For the very young children $<2$ years of age ${ }^{20}$ where allometric scaling alone might not be sufficient, a physiologically based pharmacokinetic model was utilized to recommend the starting doses ${ }^{28}$ taking into account the rapid changes in organ maturation and development in this very young population. The PK data collected from the pediatric trials were then integrated into the pediatric popPK model which was subsequently updated as PK data became available. Figure 4 shows the pathway of trials leading to the final popPK model for tapentadol OS with dose recommendations for the entire age range from birth to $<18$ years. Efficacy and safety of the thus established doses were then confirmed in a randomized placebo-controlled trial across the entire pediatric age range..$^{20,21}$

For pediatric patients requiring long-term treatment, the selection of tapentadol PR doses was based on a popPK model which integrated data from adult tapentadol PR trials and the available knowledge from pediatric tapentadol oral solution trials. Similarly, simulations with tapentadol PR aimed to identify the required doses that would result in steady-state exposures in pediatric patients similar to those reported for the therapeutic dose range of tapentadol PR in adults (50 to $250 \mathrm{mg}$ b.i.d.). The established doses were investigated in a randomized, active-controlled trial with a tapentadol PR extension phase. ${ }^{23}$

\section{Limiting Exposure in the Pediatric Population During Development is Key for Ethical and Feasibility Purposes}

The pediatric population is vulnerable and the inclusion of children in clinical trials is always a matter of great concern for parents, researchers, and ethic committees. In order to address the research questions about the effectiveness and safety of a new medicinal agent, the optimal trial 
PopPK model based on adult data

extended with allometric scaling

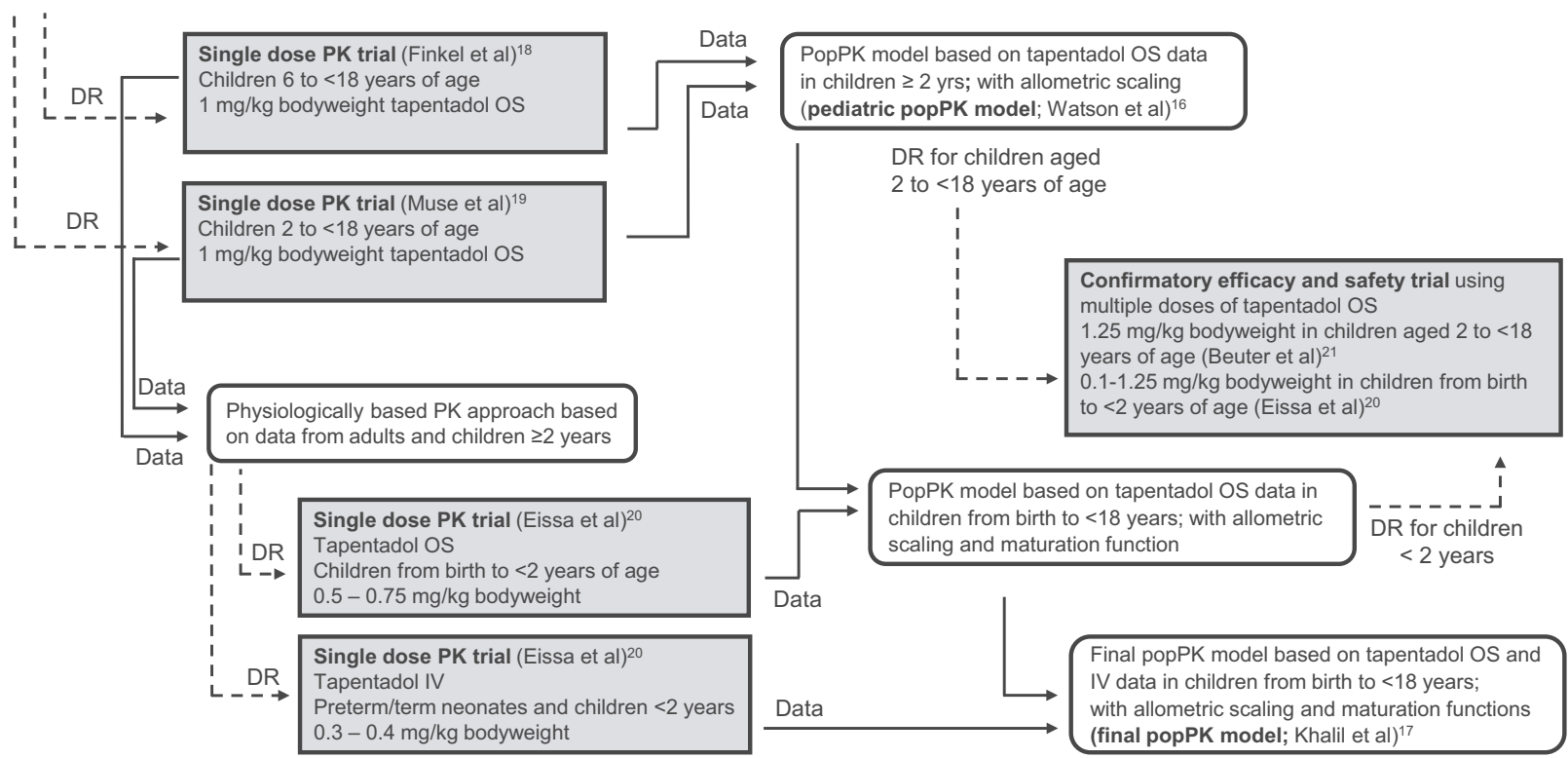

Figure 4 Dose determination pathway resulting in the final popPK model for tapentadol oral solution with dose recommendations for the entire pediatric age range from birth to $<18$ years.

Abbreviations: DR, dose recommendation; IV, intravenous infusion; OS, oral solution; popPK, population PK.

design needs to be selected to ensure that medication exposure of children is limited to the absolute minimum rendering the trial feasible while ensuring that a reliable answer to the research question can still be obtained.

In order to address the effectiveness and safety of tapentadol PR for the treatment of long-term pain in the pediatric development program, a new innovative approach developed by Hlavin et al was used for the sample size determination of the pediatric long-term pain trial. This methodology makes use of prior information and of extrapolation from a larger population to a small target population as defined in the EMA reflection paper on extrapolation. ${ }^{30}$

Specifically, for the pediatric long-term pain trial, the original sample size calculation did not consider prior information and the trial was therefore planned with a sample size of 129 children. During the trial, more information became available from the pediatric program in acute pain. Consequently, the final sample size was then recalculated making use of the pre-existing evidence from clinical trials evaluating the efficacy of tapentadol IR and PR in adult acute and chronic pain and using data from tapentadol IR in the pediatric population. By utilizing all this prior knowledge and extrapolations from adults to pediatrics and from IR to
$\mathrm{PR}$, the body of evidence required from the pediatric long-term pain trial could be reduced. This was reflected in a more liberal one-sided statistical significance level of 0.1 for the pediatric trial as determined by the Hlavin methodology, while still maintaining confidence in the effectiveness of the drug in children after successful pediatric trials. With this adjusted one-sided type I error of 0.1 , a reduced sample size of 69 children was derived, compared to 129 children required with the usual type I error of 0.025 . With this new approach, medication exposure in this vulnerable population could be limited, feasibility improved, and completion of the clinical trial accelerated.

\section{Safety is of Great Concern in Pediatric Trials and Requires Constant Monitoring}

In the tapentadol PK trials, enrollment was staggered starting with the oldest patient group under constant surveillance of safety and tapentadol exposure before the next younger group received any trial medication. In addition, external independent data monitoring committees were established for trials in children $<2$ years and for the multiple-dose efficacy and safety trial to monitor the safety of this vulnerable population. 


\section{It is Challenging to Investigate a Pain} Medication with a Broad Label in Adults in the Pediatric Population

Unlike medications indicated for a specific disorder, eg upper respiratory tract infection which can have a narrowly focused pediatric development, tapentadol with its broad authorization for acute and chronic pain indications required an extensive investigational plan in the pediatric population. In order to investigate if tapentadol could also provide effective pain relief in a broad range of pediatric pain conditions, appropriate pain conditions representative for acute and chronic pain in the pediatric population had to be identified. After extensive research, acute pain following surgery was assessed as an area of unmet need in the pediatric population with a sufficient number of patients that can be accessed to make a clinical trial feasible and as a representative model of acute pain. Two commonly used postsurgical pain models appropriate for the age group investigated (2 to $<18$ years) and associated with significant pain, namely tonsillectomy $^{31}$ and dental surgery ${ }^{32}$ were employed in one single dose PK trial ${ }^{19}$ but all other trials in the program permitted a wider selection of surgeries to achieve sufficient sample sizes and to ensure broad applicability of the results.

Typical chronic pain indications as observed in adults are rare in children. Although chronic daily pain in children might be present due to chronic headaches, neurodegenerative disorders, inflammatory disorders, posttraumatic neuropathic pain conditions, or advanced cancer and other diseases, it is less prevalent than in adults. ${ }^{33}$ Moreover, it is rare that one of these indications requires a long-term treatment with opioids in young children. In light of the low occurrence of pain requiring long-term treatment with opioids especially in young children and owing to the limitations of the tapentadol PR tablet formulations available (lowest dose available is $25 \mathrm{mg}$ ), the development program focused on patients above the age of six. Even when focusing on patients 6 years or older, it soon became clear that recruitment of a sufficient trial sample size when using the adult definition of chronic pain (ie, pain lasting for at least 3 months) would be very challenging. Moreover, discussion with experts revealed that the real need in this population is for a prolonged release formulation that can ensure adequate pain relief when use is anticipated to be longer than would be usual for the treatment of acute pain. The use of a PR formulation would allow in these cases for less frequent dosing facilitating compliance and improving quality of life for patients and caregivers. These considerations with expert input resulted in the following definition of pain for entry into the tapentadol PR trial: 'pain expected to require twice-daily PR opioid treatment for at least 14 days'. This definition was agreed by the PDCO prior to trial initiation. It permitted trial sites to recruit patients with more diverse indications (cancer pain, painful burns, postsurgical pain) in addition to patients with traditional chronic pain conditions such as juvenile rheumatoid arthritis or congenital neuropathy. ${ }^{23}$ The high proportion of patients completing 14 days of tapentadol treatment in that trial suggested that the entry criteria for severe long-term pain enabled recruitment of the appropriate patients requiring long-term treatment with a strong analgesic.

\section{Experience Gained from Interactions with the Regulatory Authorities in the EU Iterative Learning in the Course of
Development}

Tapentadol was one of the first compounds to go through the formal EU process of the Pediatric Investigation Plan (PIP) and as such had a forerunner role at the start of the learning curve for both the regulators and the pharmaceutical industry. The PIP for acute pain was agreed with PDCO in 2008 and was completed in 2018. In the course of the program, 14 modifications of this PIP were agreed. The second PIP concerning long-term pain was agreed in 2009 and is still ongoing. To date, this PIP has been modified 10 times. All modifications required the expenditure of considerable time and effort on both sides (ie PDCO and pharmaceutical company) until the modifications were agreed to and adopted. The PIP is intended to be a living document but too much detail early on necessitated this large number of changes. From the publication describing the EMA's experience with pediatric programs and the development of an ongoing action plan, it is clear that improvements have in the meantime been made and are continuing to be made in the process with less detail required initially and more flexibility to permit alteration based on emerging data; ${ }^{34,35}$ this is clearly a positive development. 


\section{Gap Between PDCO/EMA and Assessing Competent Authorities}

According to the PDCO website, "the PDCO's main role is to assess the content of paediatric investigation plans (PIPs), which determine the studies that companies must carry out in children when developing a medicine" and "is not responsible for marketing authorisation applications for medicines for use in children". This is problematic as successfully completing a PIP as agreed with the PDCO does not guarantee approval by the responsible authorities of the medicinal product for the pediatric population as intended by the PIP. The competent authorities for marketing authorization can, for example, restrict the use of the product if the program is deemed to be insufficient for the intended and unrestricted use. This can render effective use of the product in the pediatric population difficult or impossible by, eg limiting the use to single dose only or restricting use to certain settings. The ultimate goal of a pediatric development should be to make drugs available for the pediatric population. This can only be achieved when the development is in line with the valid regulatory requirements and the obtained data are reflected in the product label. In our opinion, this requires stronger involvement of regulatory experts from competent authorities in the assessment of PIPs and their modifications.

\section{Different Requirements Imposed by Different Authorities}

For tapentadol both a Pediatric Study Plan (US) and a Pediatric Investigation Plan (EU) were agreed with the respective regulatory body, FDA (US) and PDCO (EU) and subsequently executed. Unfortunately, the two plans, although largely overlapping, had some specific requirements leading to sometimes subtle or overt differences in plans between the two regions. An overt difference was the requirement in the US to conduct a placebo-controlled efficacy trial to support a pediatric long-term pain indication, whereas in the EU, a non-inferiority design was accepted. Some examples of more subtle differences include differing requirements with respect to the age ranges to be studied, eg birth to $<17$ years (not including preterm neonates) for US and birth to $<18$ years (including preterm neonates) for EU for the acute pain program. Such differences lead to increased complexity in trial design, trial conduct, and analysis and interpretation of data. This could be addressed by a closer alignment on authority requirements for pediatric programs across regions. Moreover, the lack of harmonization of national rules for conducting pediatric clinical trials across the European Union led to a further significant increase of trial duration. Some clinical trial applications for trials included in the program as agreed upon with the PDCO did not receive approval in certain EU countries due to specific local requirements. A closer upfront alignment on authority requirements for pediatric clinical trials across the $\mathrm{EU}$ would help to resolve such issues.

Recommendations for sponsors of future pediatric programs are provided (Box 1).

\section{Conclusions}

Although tapentadol is one of the latest approved opioid analgesics, it is the most thoroughly and systematically investigated strong analgesic across all age groups. Its positive benefit/risk profile that was already established in the adult population is now also supported in acute

Box I Recommendations for Sponsors of Future Pediatric Programs

- Be aware that if the initial indication obtained for adults is broad, the required program in children may be extensive.

- Engage in early discussions with the authorities PDCO and FDA to ensure that the pediatric clinical development has all chances to succeed from a scientific, ethical and feasibility perspective prior to finalization of the plan.

- Obtain agreement from competent authorities on how the outcomes of the program will be reflected in the product label prior to embarking on the pediatric development program.

- Familiarize yourself with regulations and ethical considerations in the countries where you are planning to conduct the studies.

- Put a dedicated team together that has the tenacity and stamina to overcome obstacles associated with pediatric drug development.

- Build a network of committed pediatric researchers that can help you complete the program.

- Ensure that prior to agreement on a pediatric investigational plan (EU) or pediatric study plan (FDA), its feasibility is thoroughly checked with future investigators.

- Take all prior knowledge into consideration in designing the program so that the number of patients required in the investigation will be minimized while preserving the power to obtain valid study results.

- Utilize "fit-for-purpose" modeling and simulation techniques to ensure optimal trial design and prioritize integrated data analyses especially towards the end of the program.

- Investigate if validated outcome measures are available in the age groups you want to study.

- Be critical in evaluating safety data and thoroughly assess the totality of data collected bearing in mind that for the youngest patients, verbal reporting of, eg, adverse events is not possible. 
and long-term use in the pediatric population by data from its structured pediatric development program. A pediatric development is challenging and even if executed in compliance with all preconditions from, eg a PIP, and if positive results are obtained, it may not automatically result in accessibility of the medication for the targeted population. To date and as a tangible outcome of the pediatric development plans, tapentadol OS for children has been authorized in the EU in 2018, as a new treatment option in the hospital for moderate to severe acute pain in children from 2 to $<18$ years of age.

\section{Abbreviations}

EMA, European Medicines Agency; FDA, Food and Drug Administration; IR, immediate release; IV, intravenous; MOR, $\mu$-opioid receptor agonism; NRI, noradrenaline reuptake inhibition; OS, oral solution; PDCO, Pediatric Committee; PIP, Pediatric Investigation Plan; PK, pharmacokinetic; popPK, population PK; PR, prolonged release; TEAE, treatment-emergent adverse event.

\section{Acknowledgments}

Conception and realization of the tapentadol pediatric development required the involvement of many dedicated individuals over many years. The authors would like to acknowledge everyone who contributed, in particular Maria Soledad Berdaguer, Christoph Beuter, Martin Brett, Roberta Bursi, Kevin Canales, Siak Leng Choi, Luis de la Fuente, Wieslaw Degorski, Jacqueline Delfgaauw, Jörg Diekmann, Henk Dieteren, Jens Donath, Kerry Drozda-Müller, Cecile Dubois, Alice Ebel, Jan Freijer, Jennifer Gilbride, Jutta Goldberg, Anne Gyllensvärd, Thomas Häufel, Gwyn Hopkins, Joachim Hoppmann, Uta Hössl, Andras Kasa, Lieven Kennes, Ayaz Khan, Akash Khandelwal, Tanja Küpper-Drost, Claudia Lefeber, Jiri Letal, Blanca Linares-Rivas Rico, Constanze List, Brigitte Lücking, Vishakha Oza, Corinne Pala, Wolfgang Prange, Klaus Pusecker, Jérôme Rapion, Ronald Rosenburg, Jasmina Savic, Carsten Schmidt, Achim Steup, Ariane Stollenwerk, Maria Stupar, Eva Tarau, Rolf Terlinden, Julia Tilken, Mayke van Cauteren, Gisela Volkers, Silvia von Pistor, Andrea Waßmuth, Estelle Watson, Paloma Weinrich, and Matthias Winkel. Writing and editorial assistance was provided by Elke Grosselindemann and Martin and Birgit Brett and was paid for by Grünenthal $\mathrm{GmbH}$.

\section{Disclosure}

All authors are employees of Grünenthal GmbH. The authors report no other conflicts of interest in this work.

\section{References}

1. Eerdekens M, Beuter C, Lefeber C, van den Anker J.The challenge of developing pain medications for children: therapeutic needs and future perspectives. J Pain Res. 2019;12:1649-1664. doi:10.2147/ JPR.S195788

2. Grégoire MC, Finley GA. Drugs for chronic pain in children: a commentary on clinical practice and the absence of evidence. Pain Res Manag. 2013;18(1):47-50. doi:10.1155/2013/402863

3. Califf RM Best Pharmaceuticals for Children Act and Pediatric Research Equity Act Status Report to Congress; 2016. Available from: https://www.fda.gov/downloads/scienceresearch/specialtopics/pedia trictherapeuticsresearch/ucm509815.pdf. Accessed September 8, 2020.

4. European Union. Regulation (EC) No 1901/2006 of the European Parliament and of the Council of 12 December 2006 on medicinal products for paediatric use, OJ L 378, 27.12;.2006. Available from: https://ec.europa.eu/health/sites/health/files/files/eudralex/vol-1/reg 2006_1901/reg_2006_1901_en.pdf. Accessed September 8, 2020.

5. Nafziger AN, Barkin RL. Opioid therapy in acute and chronic pain. J Clin Pharmacol. 2018;58(9):1111-1122. doi:10.1002/jcph.1276

6. Xiao JP, Li AL, Feng BM, Ye Y, Wang GJ. Efficacy and safety of tapentadol immediate release assessment in treatment of moderate to severe pain: a systematic review and meta-analysis. Pain Med. 2017;18(1):14-24. doi:10.1093/pm/pnw154

7. Viscusi E, Allard R, Sohns M, Eerdekens M. Tapentadol immediate release for moderate to severe acute post-surgery pain. $J$ Opioid Manag. 2019;15:51-67. doi:10.5055/jom.2019.0486

8. Baron R, Eberhart L, Kern KU, et al. Tapentadol prolonged release for chronic pain: a review of clinical trials and 5 years of routine clinical practice data. Pain Pract. 2017;17:678-700. doi:10.1111/papr.12515

9. Tzschentke TM, Christoph T, Kögel BY. The mu-opioid receptor agonist/noradrenaline reuptake inhibition (MOR-NRI) concept in analgesia: the case of tapentadol. CNS Drugs. 2014;28:319-329. doi:10.1007/s40263-014-0151-9

10. Raffa RB, Elling C, Tzschentke TM. Does 'strong analgesic' equal 'strong opioid'? Tapentadol and the concept of ' $\mu$-load'. Adv Ther. 2018;35:1471-1484. doi:10.1007/s12325-018-0778-x

11. Göhler K, Brett M, Smit JW, Rengelshausen J, Terlinden R. Comparative pharmacokinetics and bioavailability of tapentadol following oral administration of immediate- and prolonged-release formulations. Int $J$ Clin Pharmacol Ther. 2013;51(4):338-348. doi: $10.5414 / \mathrm{CP} 201722$

12. Terlinden R, Kogel BY, Englberger W, Tzschentke TM. In vitro and in vivo characterization of tapentadol metabolites. Methods Find Exp Clin Pharmacol. 2010;32:31-38. doi:10.1358/mf.2010.32.1.1434165

13. Tzschentke TM, De Vry J, Terlinden R, et al. Tapentadol hydrochloride. Analgesic, mu-opioid receptor agonist, noradrenaline reuptake inhibitor. Drugs Future. 2006;31:1053-1061.

14. Kneip C, Terlinden R, Beier H, Chen G. Investigations into the drug-drug interaction potential of tapentadol in human liver microsomes and fresh human hepatocytes. Drug Metab Lett. 2008;2:67-75. doi:10.2174/187231208783478434

15. Smit JW, Oh C, Rengelshausen J, et al. Effects of acetaminophen, naproxen, and acetylsalicylic acid on tapentadol pharmacokinetics: results of two randomized, open-label, crossover, drug-drug interaction studies. Pharmacotherapy. 2010;30:25-34. doi:10.1592/ phco.30.1.25

16. Watson E, Khandelwal A, Freijer J, van den Anker J, Lefeber C, Eerdekens M. Population pharmacokinetic modeling to facilitate dose selection of tapentadol in the pediatric population. J Pain Res. 2019;12:2835-2850. doi:10.2147/JPR.S208454 
17. Khalil F, Choi SL, Watson E, et al. Population pharmacokinetics of tapentadol in children from birth to $<18$ years old. J Pain Res. 2020;13:3107-3123. doi:10.2147/JPR.S269549

18. Finkel J, Goldberg J, Rosenburg R, et al. First evaluation of tapentadol oral solution for the treatment of moderate to severe acute pain in children aged 6 to <18. J Pain Res. 2019;12:1925-1936. doi:10.2147/ JPR.S197348

19. Muse D, Tarau E, Lefeber C, et al. Pharmacokinetics, safety, and efficacy of tapentadol oral solution for treating moderate to severe pain in pediatric patients. J Pain Res. 2019;12:1777-1790. doi:10.2147/JPR.S197039

20. Eissa A, Tarau E, Beuter C, et al. Tapentadol for the treatment of moderate-to-severe acute pain in children under the age of two years. $J$ Pain Res. 2021;14:229-248.

21. Beuter C, Volkers G, Radic T, Goldberg J, van den Anker J. Efficacy and safety of multiple doses of tapentadol oral solution in the treatment of moderate to severe acute pain in children aged 2 to $<18$ years - a randomized, double-blind, placebo-controlled trial. J Pain Res. 2019;12:3099-3112. doi:10.2147/JPR.S207010

22. Electronic Medicines Compendium. Palexia oral solution $20 \mathrm{mg} / \mathrm{mL}$. Available from: https://www.medicines.org.uk/emc/product/5346/ smpc. Accessed September 4, 2020.

23. Howard R, Radic T, Sohns M, Eerdekens M, Waßmuth A. Tapentadol prolonged release for long-term treatment of pain in children. J Pain Res. 2020;13:3157-3170. doi:10.2147/JPR. S272751

24. Food and Drug Administration. CDER conversations: pediatric pain management options. Available from: https:/www.fda.gov/drugs/ news-events-human-drugs/cder-conversation-pediatric-painmanagement-options. Accessed December 30, 2019.

25. Electronic Medicines Compendium. Palexia $50 \mathrm{mg}$ film-coated tablets. Available from: https://www.medicines.org.uk/emc/medi cine/28375. Accessed September 7, 2020.

26. Electronic Medicines Compendium. Palexia SR prolonged release tablets. Summary of product characteristics. Available from: https:// www.medicines.org.uk/emc/medicine/28373. Accessed September 7, 2020

27. Health Products Regulatory Authority. Palexia $4 \mathrm{mg} / \mathrm{mL}$ oral solution. Available from: https://www.hpra.ie/img/uploaded/swedocuments/ Licence_PA2242-012-010_04032020131343.pdf. Accessed September 7, 2020.
28. Khandelwal A, Bolger M, Brett M, Rosenburg R, Bursi R Physiologically-based pharmacokinetic (PBPK) model of tapentadol and tapentadol-O-glucuronide in adult and pediatric population. Available from: http://www.pkuk.org.uk/Contentimages/PKUK 2014_Programme_and_Abstractsnew.pdf. Accessed January 04, 2021.

29. Hlavin G, Koenig F, Male C, Posch M, Bauer P. Evidence, eminence and extrapolation. Stat Med. 2016;35(13):2117-2132. doi:10.1002/sim.6865

30. European Medicines Agency. Reflection paper on the use of extrapolation in the development of medicines for paediatrics. Available from: https:/www.ema.europa.eu/en/documents/scientific-guideline /adopted-reflection-paper-use-extrapolation-development-medicinespaediatrics-revision-1_en.pdf. Accessed September 7, 2020.

31. Hosseini Jahromi SA, Hosseini Valami SM, Hatamian S. Comparison between effect of lidocaine, morphine and ketamine spray on post-tonsillectomy pain in children. Anesth Pain Med. 2012;2:17-21. doi:10.5812/aapm.4092

32. Urquhart E. Analgesic agents and strategies in the dental pain model. J Dent. 1994;22:336-341. doi:10.1016/0300-5712(94)90084-1

33. Berde CB, Walco GA, Krane EJ, et al. Pediatric analgesic clinical trial designs, measures, and extrapolation: report of an FDA scientific workshop. Pediatrics. 2012;129(2):354-364. doi:10.1542/peds.20103591

34. European Medicines Agency. 10-year report to the European Commission - general report on the experience acquired as a result of the application of the Paediatric Regulation. Available from: https://ec. europa.eu/health/sites/health/files/files/paediatrics/docs/paediatrics_10 years_ema_technical_report.pdf. Accessed September 7, 2020.

35. European Medicines Agency. European Medicines Agency and European Commission (DG Health and Food Safety) action plan on paediatrics. Available from: https:/www.ema.europa.eu/en/documents/report/eur opean-medicines-agency-european-commission-dg-health-food-safetyaction-plan-paediatrics_en.pdf. Accessed September 8, 2020.

36. Zannikos PN, Smit JW, Stahlberg HJ, Wenge B, Hillewaert VM, Etropolski MS. Pharmacokinetic evaluation of tapentadol extended-release tablets in healthy subjects. J Opioid Manag. 2013;9(4):291-300. doi:10.5055/jom.2013.0171
Journal of Pain Research

\section{Publish your work in this journal}

The Journal of Pain Research is an international, peer reviewed, open access, online journal that welcomes laboratory and clinical findings in the fields of pain research and the prevention and management of pain. Original research, reviews, symposium reports, hypothesis formation and commentaries are all considered for publication. The manuscript

Submit your manuscript here: https://www.dovepress.com/journal-of-pain-research-journal management system is completely online and includes a very quick and fair peer-review system, which is all easy to use. Visit http:// www.dovepress.com/testimonials.php to read real quotes from published authors. 